International Mathematical Forum, 2, 2007, no. 19, 905 - 909

\title{
The Maps Preserving Approximation
}

\author{
H. Mazaheri and M. Hossein Zadeh \\ Department of Mathematics \\ Yazd University, Yazd, Iran \\ hmazaheri@yazduni.ac.ir
}

\begin{abstract}
The purpose of this paper is to introduce and discuss the concept of the maps which preserve approximation. We show that if a operator on a normed space is an isometry then preserves all approximation property, every linear operator preserving approximation is an isometry multiplied by a constant.
\end{abstract}

Mathematics Subject Classification: 41A65, 46B50, 46B20, 41A50

Keywords: Approximation, Preserve approximation, Orthogonal subspace, Chebyshev subspaces, Proximinal space

\section{Introduction}

Suppose $X$ is a normed linear space and $x, y \in X, x$ is said to be orthogonal to $y$ and is denoted by $x \perp y$ if and only if $\|x\| \leq\|x+\alpha y\|$ for all scalar $\alpha$. If $G_{1}$ and $G_{2}$ are subsets of $X$, it is defined $G_{1} \perp G_{2}$ if and only if for all $g_{1} \in G_{1}, g_{2} \in G_{2}, g_{1} \perp g_{2}$. (see [1]). Let $G$ be a subspace of $X$, it is defined the set of the metric complemented

$$
\hat{G}=\{x \in X: \quad x \perp G\}=\{x \in X:\|x\|=\|x+G\|,
$$

and the set of the cometric complement

$$
\breve{G}=\{x \in X: G \perp x\} .
$$

We know that a point $g_{0} \in G$ is said to be a best approximation (resp. best coapproximation) for $x \in X$ if and only if $\left\|x-g_{0}\right\|=\|x+G\|=$ $\operatorname{dist}(x, G)$ (resp. $\left\|g_{0}-g\right\| \leq\|x-g\| \forall g \in G$ ). It can be easily proved that $g_{0}$ is a best approximation (resp. best coapproximation) for $x \in X$ if and only if $x-g_{0} \in \hat{G}$ (resp. $x-g_{0} \in \breve{G}$ ). The set of all best approximations (resp. 
best coapproximations) of $x \in X$ in $G$ is shown by $P_{G}(x)\left(\operatorname{resp} \cdot R_{G}(x)\right)$. In other words,

$$
P_{G}(x)=\left\{g_{0} \in G: x-g_{0} \in \hat{G}\right\}
$$

and

$$
R_{G}(x)=\left\{g_{0} \in G: x-g_{0} \in \breve{G}\right\} .
$$

If $P_{G}(x)\left(\operatorname{resp} . R_{G}(x)\right)$ is non-empty for every $x \in X$, then $G$ is called an Proximinal (resp. coproximinal) set. The set $M$ is Chebyshev (resp. cochebyshev) if $P_{G}(x)\left(\right.$ resp. $\left.R_{G}(x)\right)$ is a singleton set for every $x \in X$. (see [5-13])

A proximinal subspace $G$ is called quasi-Chebyshev if and only if $P_{G}(x)$ is compact, for all $x \in X$ (see [3-4]).

Suppose $G$ is a subspace of $X$ then $G$ is called orthogonal complemented subspace, if either $G$ is Chebyshev and $\hat{G}$ is a subspace of $X$ or $G$ is cochebyshev and $\breve{G}$ is a subspace of $X$.

\section{Preserves approximation}

In this section we shall obtain characterization of preserving approximation maps.

Lemma 2.1. Let $X$ be a normed linear space. If $T: X \rightarrow X$ is an isometry operator, then for all subspace $G$ of $X$ and all $x \in X$,

$$
T\left(P_{G}(x)\right)=P_{T(G)}(T(x)) \text { and } T\left(R_{G}(x)\right)=R_{T(G)}(T(x)) .
$$

Proof. We have for all $x \in X,\|T(x)\|=\|x\|$, therefore for all $x \in X$ and all subspace $G$ of $X$ and $g, g_{0} \in G$,

$$
\left\|x-g_{0}\right\| \leq\|x-g\| \Leftrightarrow\left\|T(x)-T\left(g_{0}\right)\right\| \leq\|T(x)-T(g)\| .
$$

Definition 2.2. Suppose $X, Y$ are two linear normed spaces. A map $T: X \rightarrow$ $Y$ is called preserving approximation (resp. preserving coapproximation) if and only if for all subspace $G$ of $X$ and all $x \in X$,

$$
T\left(P_{G}(x)\right)=P_{T(G)}(T(x))\left(\text { resp. } T\left(R_{G}(x)\right)=R_{T(G)}(T(x)) .\right.
$$

Corollary 2.3. Let $X$ be a normed linear space. Every isometry operator $T: X \rightarrow X$ is preserving approximation (resp. preserving coapproximation).

Theorem 2.4. Suppose $X, Y$ are two linear normed spaces and $T: X \rightarrow Y$ is a linear map which is preserves approximation (resp. preserves coapproximation).

a) Suppose $G$ is a subspace of $X$, then $G$ is proximinal (resp. coproximinal) of $X$, if and only if $T(G)$ is proximinal (resp. coproximinal) of $Y$. 
b) Suppose $G$ is a subspace of $X$, then $G$ is Chebyshev (resp. cochebyshev) if and only if $T(G)$ is Chebyshev (resp. cochebyshev.

c) If $T$ is linear, then $\forall x, y \in X \quad x \perp y \Longrightarrow T(x) \perp T(y)$. (T is called preserving orthogonality).

d) For a subspace $G$ of $X, T(\widehat{G})=\widehat{T(G)}(\operatorname{resp} . T(\breve{G})=T \breve{T(G)})$.

e) Suppose $G$ is a subspace of $X$, then $G$ is orthogonality complemented in $X$ if and only if $T(G)$ is orthogonality complemented in $Y$.

f) Suppose $G$ is a subspace of $X$, if $T$ is a cotinuous and onto preserves approximation map, then $G$ is quasi chebyshev if and only if $T(G)$ is quasi Chebyshev.

Proof. We can easily prove (a), (b), (d), (e). We prove the parts (c) and (f).

c) Suppose $T$ is a preserves approximation, if $x, y \in X$ and $x \perp y$, then $0 \in P_{<y>}(x)$. Therefore $0=T(0) \in P_{T(<y>)}(T(x))$, since $T$ is linear, $T(<y>$ )$=<T(y)>$. Hence $T(x) \perp T(y)$

Now suppose $T$ is a preserves approximation, if $x, y \in X$ and $x \perp y$, then $0 \in R_{<x>}(y)$. Therefore $0=T(0) \in R_{T(<x>)}(T(y))$, since $T$ is linear, $T(<x>$ )$=<T(x)>$. Hence $T(x) \perp T(y)$.

f) Let $z \in Y$ and $\left\{u_{n}\right\} \subseteq P_{T(G)}(z)$. Since $T$ is onto, we have $z=T(x)$ for some $x \in X$, therefore $\left\{u_{n}\right\} \subseteq T\left(P_{G}(x)\right)$. Then there exists a sequence $\left\{v_{n}\right\} \subseteq P_{G}(x)$ such that $u_{n}=T\left(v_{n}\right)$. Since $G$ is quasi Chebyshev, the set $P_{G}(x)$ is compact. Hence there exists subsequence $\left\{v_{n_{k}}\right\}_{k \geq 1}$ of $\left\{v_{n}\right\}$ and $v_{0} \in X$ such that $v_{n_{k}} \rightarrow v_{0}$. Sinve $T$ is continuous, $u_{n_{k}}=T\left(v_{n_{k}}\right) \rightarrow u_{0}=T\left(v_{0}\right)$. Therefore $\left.P_{T(G)}(z)\right)$ is compact.

Theorem 2.5. Let $X$ be a normed linear space and the operator $T: X \rightarrow X$ be preserving approximation, then $T=k U$ such that $k \in R$ and $U$ is an isometry.

Proof. We know that $T$ is preserving approximation, from Theorem 2.4, $T$ is preserving orthogonality. From [1], $T$ is an isometry multiplied by a constant.

Example 2.6. There exists non-linear map which it is preserving orthogonality. We define $T: R^{2} \longrightarrow R^{2}$ by $T(x, y)=(x, y)$ if $x, y \neq 0, T(x, y)=(1,1)$ if $y=0, x \neq 0, T(x, y)=(-1,1)$ if $x=0, y \neq 0$ and $T(0,0)=(0,0)$.

\section{3. $\epsilon$-Preserves approximation}

In this section we shall obtain characterization of $\epsilon$-Preserves approximation maps. Let $X$ be a normed linear space $\epsilon>0$ and $x, y \in X$. We call $x$ is $\epsilon$ orthogonal to $y$ and is denoted by $x \perp_{\epsilon} y$ if and only if $\|x\| \leq\|x+\alpha y\|+\epsilon$ for all scalar $\alpha$. If $G_{1}$ and $G_{2}$ are subsets of $X$, we define $G_{1} \perp_{\epsilon} G_{2}$ if and only if for all $g_{1} \in G_{1}$ and $g_{2} \in G_{2}$ we have, $g_{1} \perp_{\epsilon} g_{2}$. 
For $\epsilon>0$ we have,

$$
\widehat{G_{\epsilon}}=\left\{x \in X: \quad x \perp_{\epsilon} G\right\} .
$$

For $\epsilon>0$, a point $g_{0} \in G$ is said to be a $\epsilon$-approximation for $x \in X$ if $x-g_{0} \in \widehat{G}_{\epsilon}$. The set of all $\epsilon$-approximation for $x \in X$ be denoted by $P_{G, \epsilon}(x)$. Also we have

$$
P_{G, \epsilon}(x)=\left\{g_{0} \in G:\left\|x-g_{0}\right\| \leq\|x-g\|+\epsilon \text { for all } g \in G\right\} .
$$

For all $\epsilon>0$, it is clear that the set $P_{G, \epsilon}(x)$ is a nonempty set. for more information see [6] and [11].

Definition 3.1. Suppose $X, Y$ are two linear normed spaces and $\epsilon>0$. A map $T: X \rightarrow Y$ is called $\epsilon$-preserving approximation if and only if for all subspace $G$ of $X$ and all $x \in X$,

$$
T\left(P_{G, \epsilon}(x)\right)=P_{T(G), \epsilon}(T(x)) .
$$

Corollary 3.2. Let $X$ be a normed linear space and $\epsilon>0$. Then every isometry operator $T: X \rightarrow X$ be $\epsilon$-preserving approximation.

Theorem 3.3. Suppose $X, Y$ are two linear normed spaces, $\epsilon>0$ and $T$ : $X \rightarrow Y$ is an onto which is $\epsilon$-preserves approximation.

c) If $T$ is linear, then $\forall x, y \in X \quad x \perp_{\epsilon} y \Longrightarrow T(x) \perp_{\epsilon} T(y)$.

d) For a subspace $G$ of $\left.X, T\left(\widehat{G_{\epsilon}}\right)=T \widehat{T(G}\right)_{\epsilon}$.

\section{References}

[1] A. Koldobsky, Operators preserving orthogonality are isometries, Submitted.

[2] G. Brikhoff, Orthogonality in linear metric space, Duke Math. J. 1(1935) 169-172.

[3] C. Franchetti, M. Furi, Some characteristic properties of real Hilbert spaces, Rwy Romaine Math., 1972.

[4] F. Mazaheri, F. M. Maalek Ghaini, Quasi-orthogonality of the best approximant sets, Nonlinear Analysis, 65(2006), no. 3, 534-537.

[5] H. Mazaheri, S. M. Modarress, Some results concerning proximinality and Coproximinality, Nonlinear Analysis, 62(2005) no. 6, 1123-1126.

[6] H. Mazaheri, S. M. Vaezpour, Orthogonality and $\epsilon$-Orthogonality in Banach spaces, Aust. J. Math. Anal. Appl., 2 (2005) no. 1, Art. 10, 1-5.

[7] T. D. Narang, Best coapproximation in metric spaces, Publ. Inst. math. (Beograd) (N.S) 51(65)(1992) 71-76. 
[8] P. L. Papini, I. Singer, Best coapproximation in normed linear spaces, Mh. Math., 88(1979) 27-44.

[9] G. S. Rao, K. R. Chandrasekaran, Best coapproximation in normed linear spaces with property $(\Lambda)$, Math. Today, 2(1984) 33-40.

[10] G. S. Rao, K. R. Chandrasekaran, Some properties of the maps $R_{G}$ and $R_{G}$, Pure Appl. Math. Sci., 23(1986) 21-27.

[11] G. S. Rao, K. R. Chandrasekaran, Characterization of elements of best coapproximation in normed linear spaces, Pure Appl. Math. Sci., 26(1987) 139-147.

[12] G. S. Rao, R. Saravanan, Some results concerning best uniform coapproximation., Journal of Inequal. in pure and Appl. Math., 3(2002) no. 2, 1-13.

[13] G. S. Rao, R. Saravanan, Strongly unique best coapproximation, Kyungpook math. J., 43(2003) no. 4, 519-538.

[14] I. Singer, Best approximation in normal linear spaces by elements of linear subspaces, Springer, New York 1970.

Received: July 6, 2006 